\title{
MEASURE VALUED SOLUTIONS FOR SYSTEMS GOVERNED BY NEUTRAL DIFFERENTIAL EQUATIONS ON BANACH SPACES AND THEIR OPTIMAL CONTROL
}

\author{
N.U. AHMED \\ University of Ottawa
}

\begin{abstract}
In this paper we consider the question of existence of measure valued solutions for neutral differential equations on Banach spaces when there is no mild solutions. We prove the existence of measure solutions and their regularity properties. We consider also control problems of such systems and prove existence of optimal feedback controls for some interesting a-typical control problems.
\end{abstract}

Keywords: neutral differential equations, Banach spaces, optimal control. 2010 Mathematics Subject Classification: 49J27, 60H15,93E20.

\section{INTRODUCTION}

Let $E$ be a Banach space and consider the classical evolution equation on $E$ given by

$$
(d / d t) x=A x+f(t, x), x(0)=x_{0} \in E, t \in I \equiv[0, T], T<\infty,
$$

where $A$ is the infinitesimal generator of a $C_{0}$-semigroup $S(t), t \geq 0$, in $E$, and the vector field $f: I \times E \longrightarrow E$ is continuous and bounded on bounded sets. It is well known that if the vector field is merely continuous and even uniformly bounded the equation (1) may have no solution even in the mild sense. There are interesting counter examples given by Godunov [13] and Dieudonné [15]. In recent years there has been great interest in the study of measure valued solutions for this class of systems and their various generalizations including stochastic systems and systems driven by vector measures [1-11]. According to the authors knowledge, it seems there has been no attempt at considering measure valued solutions for neutral systems governed by evolution equations of the form:

$$
(d / d t)(x+g(t, x))=A x+f(t, x), x(0)=x_{0} \in E, t \in I \equiv[0, T], T<\infty,
$$


where $A$ and $f$ are as described for the system (1), and the map $g: I \times E \longrightarrow E$ is continuous and bounded on bounded sets. Neutral systems include, among other topics, systems governed by parabolic partial differential equations with nonhomogeneous boundary data. For details the reader is referred to [18, Part. 1] and [19, Chapter 3] and the references therein. In this paper we consider the question of existence of measure valued solutions for this class of systems and also consider their feedback control.

The rest of the paper is organized as follows. In Section 2, we introduce some basic background materials required for the study of measure valued solutions of the above system. In Section 3, questions of existence and regularity of measure solutions are considered in details. In Section 4, we consider the question of continuous dependence of solutions on feedback controls in certain weak topologies and then study several interesting standard and nonstandard control problems addressing the questions of existence of optimal controls.

\section{BASIC BACKGROUND MATERIALS}

Let $X$ be any regular topological space and $B C(X)$ the Banach space of bounded continuous functions endowed with supnorm topology. It is well known that the topological dual of this space is given by $\mathcal{M}_{r b a}(X)$, the space of regular bounded finitely additive measures. The later space endowed with total variation norm is a Banach space.

Lemma 2.1 [12]. The topological dual $B C(X)^{*}$ is isometrically isomorphic to $\mathcal{M}_{r b a}(X)$ in the sense that for any $\ell \in B C(X)^{*}$ there exists a unique element $\mu \in \mathcal{M}_{r b a}(X)$ such that

$$
\ell(\varphi)=\int_{X} \varphi(x) \mu(d x)
$$

and that $\|\ell\|=|\mu|_{v}$.

We are interested in the space of regular bounded finitely additive probability measures on $X$ which is denoted by $\Pi_{r b a}(X)$. Clearly this is a subset of $\mathcal{M}_{r b a}(X)$. Further on we need the concept of measure valued function $\mu: I \equiv[0, T] \longrightarrow$ $\mathcal{M}_{r b a}(X)$. It is well known that the spaces $B C(X)$ and $\mathcal{M}_{r b a}(X)$ do not satisfy the RNP (Radon-Nikodym property). Hence the dual of $L_{1}(I, B C(X))$ is not given by $L_{\infty}\left(I, \mathcal{M}_{r b a}(X)\right)$. However by virtue of the theory of lifting, the (topological) dual of $L_{1}(I, B C(X))$ is given by $L_{\infty}^{w}\left(I, \mathcal{M}_{r b a}(X)\right)$ which consists of weak star measurable $\mathcal{M}_{r b a}(X)$ valued functions. Any continuous linear functional $\ell$ on $L_{1}(I, B C(X))$ has the representation

$$
\ell(\varphi)=\int_{I \times X} \varphi(t, x) \mu_{t}(d x) d t
$$


for some $\mu \in L_{\infty}^{w}\left(I, \mathcal{M}_{r b a}(X)\right)$.

In this work we need the set $L_{\infty}^{w}\left(I, \Pi_{r b a}(X)\right) \subset L_{\infty}^{w}\left(I, \mathcal{M}_{r b a}(X)\right)$. Clearly, endowed with the weak star topology, it follows from Alaoglu's theorem that this is a compact set.

Definition 2.2. A measure valued function $\mu \in L_{\infty}^{w}\left(I, \Pi_{r b a}(E)\right)$ is said to be a measure solution of the evolution equation (1) if and only if it satisfies the following identity

$$
\mu_{t}(\varphi)=\varphi\left(x_{0}\right)+\int_{0}^{t} \mu_{t}(\mathcal{A} \varphi) d s, t \in I,
$$

for each $\varphi \in D(\mathcal{A})$ where the operator $\mathcal{A}$ is given by

$$
(\mathcal{A} \varphi)(t, \xi) \equiv<A^{*} D \varphi(\xi), \xi>_{E^{*}, E}+<D \varphi(\xi), f(t, \xi)>_{E^{*}, E} .
$$

\section{EXISTENCE OF MEASURE SOLUTIONS AND THEIR REGULARITY}

Let us consider the evolution equation (2). For simplicity we assume that $g$ is independent of $t$ though it works also for time varying $g$. This assumption is used only for the following result. Later in the sequel we use more relaxed assumptions as given in Theorem 3.4.

Theorem 3.1. Consider the system (2) and suppose that

(i) $A$ is the infinitesimal generator of a $C_{0}$-semigroup $S(t), t \geq 0$, on $E$,

(ii) $f: I \times E \longrightarrow E$ is continuous and bounded on bounded sets satisfying the following approximation properties:

(a1) there exists a sequence $\left\{f_{n}\right\}$ such that $f_{n}(t, x) \in D(A)$ for $x \in E$ and $t \in I$ and further, for each $e^{*} \in E^{*}$,

$$
<e^{*}, f_{n}(t, x)>_{E^{*}, E} \longrightarrow<e^{*}, f(t, x)>_{E^{*}, E}, \text { for each } x \in E, t \in I,
$$

as $n \rightarrow \infty$.

(a2) there exists a pair $\left\{\alpha_{n}, \beta_{n}\right\} \in L_{1}^{+}(I)$, possibly $\left\|\alpha_{n}\right\|_{L_{1}},\left\|\beta_{n}\right\|_{L_{1}} \longrightarrow \infty$, so that $\left|f_{n}(t, x)-f_{n}(t, y)\right|_{E} \leq \alpha_{n}(t)|x-y|_{E}$ and $\left|f_{n}(t, x)\right|_{E} \leq \beta_{n}(t)$ $\left(1+|x|_{E}\right\}$ for all $x, y \in E$.

(iii) $g: E \longrightarrow E$ is Lipschitz with Lipschitz constant $0 \leq K<1$.

Then for every initial state $x_{0} \in E$ (or initial measure $\mu_{0}=\nu$ ) the system 


$$
(d / d t)(x+g(x))=A x+f(t, x), x(0)=x_{0} \in E, t \in I \equiv[0, T], T<\infty,
$$

has at least one measure valued solution $\mu \in L_{\infty}^{w}\left(I, \Pi_{r b a}(E)\right)$.

Proof. We present a brief outline of the proof based on our previous results for standard evolution equations such as equation (1) [2]. Consider the algebraic equation $y=x+g(x)$ on the Banach space $E$. For any $y \in E$, introduce the operator $G_{y}(x) \equiv y-g(x)$. It is easy to see that for each $y \in E$, the operator $G_{y}$ is Lipschitz with Lipschitz constant $K(<1)$ and hence by Banach fixed point theorem $G_{y}$ has a unique fixed point $x \in E$. This defines the resolvent operator $H: E \longrightarrow E$ given by $x=H(y)$. The reader can easily verify that the resolvent operator $H$ is also Lipschitz with Lipschitz constant $1 /(1-K)$. Thus we can reformulate the original problem given by equation (6) in the following form

$$
\begin{aligned}
(d / d t)(y) & =A H(y)+f(t, H(y)), t \in I \equiv[0, T], T<\infty, \\
y(0) & =y_{0} \equiv x_{0}+g\left(x_{0}\right) \in E .
\end{aligned}
$$

We prove that equation (7) has a measure valued solution. For this we introduce the space of test functions

$$
\Phi \equiv\left\{\varphi \in B C(E): D \phi \in B\left(E, E^{*}\right)\right\}
$$

and the operator $\mathcal{A}$ given by

$$
D(\mathcal{A}) \equiv\{\varphi \in \Phi: \mathcal{A} \varphi \in B C(E)\}
$$

where

$$
(\mathcal{A} \varphi)(t, \xi)=<A^{*} D \varphi(\xi), H(\xi)>_{E^{*}, E}+<D \varphi(\xi), f(t, H(\xi))>_{E^{*}, E} .
$$

Since $H$ is Lipschitz and $(t, x) \longrightarrow f(t, x)$ is continuous on $I \times E$, the composition map $\tilde{f}(t, y) \equiv f(t, H(y))$ is continuous and bounded on bounded sets. By use of Yosida approximation, set $H_{n}(e)=n R(n, A) H(e), e \in E$ and $y_{0, n}=n R(n, A) y_{0}$ for $n \in \rho(A)$, the resolvent set of $A$. Then under the given assumptions all the conditions of Theorem 3.2 [2, Theorem 3.2, p. 12] are satisfied. Hence it follows from this theorem that there exists at least one measure valued function $\mu \in$ $L_{\infty}^{w}\left(I, \Pi_{r b a}(E)\right)$ satisfying

$$
\mu_{t}(\varphi)=\varphi\left(y_{0}\right)+\int_{0}^{t} \mu_{s}(\mathcal{A} \varphi) d s, t \in I, \forall \varphi \in D(\mathcal{A})
$$

and therefore, by definition $\mu$ is a measure valued solution for the evolution equation (7). Let this solution be denoted by $\mu^{(7)}$. We use this measure solution 
to determine the existence of a measure solution for the original problem (6) which we denote by $\mu^{(6)}$. Clearly it follows from the algebraic relation $y=x+g(x)$ that the measure $\mu^{(6)}$ is related to the measure $\mu^{(7)}$ as follows

$$
\int_{E} \varphi(\eta) \mu_{t}^{(7)}(d \eta)=\int_{E} \varphi(\xi+g(\xi)) \mu_{t}^{(6)}(d \xi)
$$

for every $\varphi \in B C(E)$ and $t \in I$. Clearly, the relation (10) prescribes and characterizes the measure solution $\mu^{(6)} \in L_{\infty}^{w}\left(I, \Pi_{r b a}(E)\right)$ in terms of the measure valued solution $\mu^{(7)}$. An alternate relation, based on the transformation $x=H(y)$, is given by

$$
\int_{E} \varphi(\xi) \mu_{t}^{(6)}(d \xi)=\int_{E} \varphi(H(\xi)) \mu_{t}^{(7)}(d \xi)
$$

for every $\varphi \in B C(E)$ and $t \in I$. Thus we have proved the existence of a measure solution for the original problem (6) given by $\mu^{(6)} \in L_{\infty}^{w}\left(I, \Pi_{r b a}(E)\right)$. This completes the brief outline of the proof.

Remark 3.2. Let $\mu^{\zeta}$ denote the solution of equation (9) for $y_{0}=\zeta \in E$. In case the measure induced by the initial state $y_{0}$ is given by $\nu$, the equation (9) is replaced by

$$
\hat{\mu}_{t}(\varphi)=\nu(\varphi)+\int_{0}^{t} \hat{\mu}_{s}(\mathcal{A} \varphi) d s, t \in I
$$

where now the solution $\hat{\mu}$ is given by the convolution $\hat{\mu}_{t}(\varphi)=\int_{E} \mu_{t}^{\zeta}(\varphi) \nu(d \zeta)$ for $\varphi \in B C(E)$.

The method used above for proof of existence of measure solution is very powerful. It applies to semilinear, quasilinear and nonlinear problems without requiring $A$ to be the infinitesimal generator of a $C_{0}$-semigroup [2-11]. However, for neutral systems, the assumption that the map $g$ is a contraction, is a limitation. So we may use another technique which works very well for semilinear problems provided $A$ generates a $C_{0}$-semigroup. The technique that we are going to use now is known as the method of transposition. This is the technique used in $[2$, Theorem 2.2, p. 8$]$. Let $S(t), t \geq 0$, denote the semigroup generated by $A$, we can formally use Dhumels formula and integration by parts to convert the differential equation (2) into the following integral equation

$$
\begin{aligned}
x(t)+g(t, x(t))= & S(t)\left(x_{0}+g\left(0 . x_{0}\right)\right)+\int_{0}^{t} S(t-r) f(r, x(r)) d r \\
& -\int_{0}^{t} A S(t-r) g(r, x(r)) d r .
\end{aligned}
$$


Since $f$ and $g$ are assumed to be merely continuous and bounded on bounded sets this integral equation may have no mild solution. Thus the identity is formal. However under fairly general assumptions, we can prove the existence of measure valued solutions. For this we need the following definition.

For each $r \in[0, \infty)$, let $B_{r}=B_{r}(E)$ denote the closed ball in $E$ of radius $r$ centered at the origin.

Definition 3.3. The evolution equation (2) is said to have a sign indefinite measure valued solution if, for each $\mu_{0} \in M_{r b a}(E)$, there exists a $\mu \in L_{\infty}^{w}\left(I, M_{r b a}(E)\right)$ such that for each $e^{*} \in D\left(A^{*}\right) \subset E^{*}$ and $r \in[0, \infty), \mu$ satisfies the following functional equation in $L_{\infty}^{w}\left(I, M_{r b a}(E)\right)$

$$
\begin{aligned}
\int_{B_{r}}<\xi+g & (t, \xi), e^{*}>_{E, E^{*}} \mu_{t}(d \xi)=\int_{B_{r}}<S^{*}(t) e^{*}, \xi+g(0, \xi)>\mu_{0}(d \xi) \\
& +\int_{0}^{t} \int_{B_{r}}<S^{*}(t-s) e^{*}, f(s, \xi)>\mu_{s}(d \xi) d s \\
& -\int_{0}^{t} \int_{B_{r}}<S^{*}(t-s) A^{*} e^{*}, g(s, \xi)>\mu_{s}(d \xi) d s, t \in I .
\end{aligned}
$$

And it is said to have a (probability) measure valued solution, if $\mu \in$ $L_{\infty}^{w}\left(I, \Pi_{r b a}(E)\right)$ for $\mu_{0} \in \Pi_{r b a}(E)$.

Theorem 3.4. Consider the evolution equation (2) and suppose $A$ generates a $C_{0}$-semigroup $S(t), t \geq 0$, in $E$ and $g, f: I \times E \longrightarrow E$ are continuous and bounded on bounded sets of $I \times E$. Then, for any initial measure $\mu_{0}=\nu \in M_{r b a}(E)$, the system (2) has at least one sign indefinite measure solution $\mu \in L_{\infty}^{w}\left(I, M_{r b a}(E)\right)$ in the sense of Definition 3.3.

Proof. For any $r \in[0, \infty)$ and any given initial state $\mu_{0}=\nu \in M_{r b a}(E)$, define the function

$$
h_{r}(t) \equiv \int_{B_{r}}<S^{*}(t) e^{*}, \xi+g(0, \xi)>_{E^{*}, E} \nu(d \xi), t \in I .
$$

Since $S^{*}(t), t \geq 0$, is weak star continuous, it follows from the assumption on $g$ that the integrand is continuous and bounded on $I \times B_{r}(E)$ for every finite $r \geq 0$. Hence $h_{r} \in L_{\infty}(I)$. Introduce the operator $L_{r} \equiv L_{r, 1}+L_{r, 2}+L_{r, 3}$ where

$$
\begin{aligned}
& L_{r, 1}(\mu)(t) \equiv \int_{B_{r}}<\xi+g(t, \xi), e^{*}>_{E, E^{*}} \mu_{t}(d \xi), t \in I, \\
& L_{r, 2}(\mu)(t) \equiv \int_{0}^{t} \int_{B_{r}}<S^{*}(t-s) A^{*} e^{*}, g(s, \xi)>\mu_{s}(d \xi) d s, t \in I, \\
& L_{r, 3}(\mu)(t) \equiv-\int_{0}^{t} \int_{B_{r}}<S^{*}(t-s) e^{*}, f(s, \xi)>\mu_{s}(d \xi) d s, t \in I .
\end{aligned}
$$


Again, under the assumptions on $g$ and $f$, it is clear that the operators $\left\{L_{r, i}\right.$, $i=1,2,3\}$ map $L_{\infty}^{w}\left(I, M_{r b a}\left(B_{r}(E)\right)\right)$ to $L_{\infty}(I)$ and hence, for each $r \in[0, \infty)$, the map $L_{r}$ is a bounded linear operator from $L_{\infty}^{w}\left(I, M_{r b a}\left(B_{r}(E)\right)\right)$ to $L_{\infty}(I)$. Clearly, equation (14) can be written in the compact form

$$
L_{r} \mu=h_{r} .
$$

Our problem is to prove the existence of a solution of this equation in the space $L_{\infty}^{w}\left(I, M_{r b a}\left(B_{r}(E)\right)\right)$ for every $h_{r} \in L_{\infty}(I)$ corresponding to the initial measure $\mu_{0}=\nu \in M_{r b a}\left(B_{r}(E)\right)$. We use the method of transposition as in [2]. Using the adjoint of the operator $L_{r}$ we can construct an isomorphism as follows. First note that the adjoint operators corresponding to the operators $\left\{L_{r, i}\right\}$ are given by

$$
\begin{aligned}
& \left(L_{r, 1}^{*} \eta\right)(t, \xi) \equiv<\xi+g(t, \xi), e^{*}>\eta(t),(t, \xi) \in I \times B_{r}(E) \\
& \left(L_{r, 2}^{*} \eta\right)(t, \xi) \equiv \int_{t}^{T}<S^{*}(s-t) A^{*} e^{*}, g(t, \xi)>\eta(s) d s,(t, \xi) \in I \times B_{r}(E), \\
& \left(L_{r, 3}^{*} \eta\right)(t, \xi) \equiv-\int_{t}^{T}<S^{*}(s-t) e^{*}, f(t, \xi)>\eta(s) d s,(t, \xi) \in I \times B_{r}(E) .
\end{aligned}
$$

It is clear from the above expressions that for every $\eta \in L_{1}(I)$, we have $\left(L_{r, i}^{*} \eta\right) \in$ $L_{1}\left(I, B C\left(B_{r}(E)\right)\right)$ for each finite $r>0$ and for each $i=1,2,3$. Hence $L_{r}^{*}$ : $L_{1}(I) \longrightarrow L_{1}\left(I, B C\left(B_{r}(E)\right)\right)$ for every finite $r>0$ where $L_{r}^{*}=L_{r, 1}^{*}+L_{r, 2}^{*}+L_{r, 3}^{*}$. Now we introduce the space

$$
Y_{r} \equiv\left\{y \in L_{1}\left(I, B C\left(B_{r}(E)\right)\right): y=L_{r}^{*} \eta \text { for some } \eta \in L_{1}(I)\right\}
$$

and endow this with the norm topology given by:

$$
\|y\|_{Y_{r}} \equiv \sup \left\{\|\eta\|_{L_{1}(I)}: y=L_{r}^{*} \eta\right\}
$$

The reader can easily verify that $Y_{r}$ is a normed vector space and that with respect to this norm topology it is a Banach space. Clearly, it follows from the definition of the set $Y_{r}$, that $L_{r}^{*}$ is surjective, that is, $L_{r}^{*}\left(L_{1}(I)\right)=Y_{r}$. Further, note that for every $\eta \in L_{1}(I)$ we have the inequality

$$
\left\|L_{r}^{*} \eta\right\|_{Y_{r}} \geq\|\eta\|_{L_{1}(I)}, \forall \eta \in L_{1}(I)
$$

and so $L_{r}^{*}$ is injective. Thus $L_{r}^{*}$ is a bijective map between the spaces $L_{1}(I)$ and $Y_{r}$ and hence $L_{r}^{*} \in \mathcal{I} s o\left(L_{1}(I), Y_{r}\right)$. For the given $h_{r} \in L_{\infty}(I)$, it is clear that the map $\ell_{r}(\eta) \equiv\left(h_{r}, \eta\right)_{L_{\infty}, L_{1}}=\int_{I} h_{r}(t) \eta(t) d t$ defines a continuous linear functional on the space $L_{1}(I)$. Since $L_{r}^{*} \in \mathcal{I} s o\left(L_{1}, Y_{r}\right)$, this linear functional on $L_{1}(I)$ is 
equivalent to the linear functional

$$
\tilde{\ell}_{r}(y) \equiv \ell_{r}\left(\left(L_{r}^{*}\right)^{-1}(y)\right) \equiv\left(\ell_{r} o\left(L_{r}^{*}\right)^{-1}\right)(y) \text { on } Y_{r}
$$

and it follows from (24) that it is a continuous linear functional on $Y_{r}$. Hence there exists a $y_{o}^{*} \in Y_{r}^{*} \subset L_{\infty}^{w}\left(I, M_{r b a}\left(B_{r}(E)\right)\right.$ ) (dual of $\left.Y_{r}\right)$ such that

$$
\tilde{\ell}_{r}(y)=<y_{o}^{*}, y>_{Y_{r}^{*}, Y_{r}} \equiv y_{o}^{*}(y)
$$

for all $y \in Y_{r}$. It follows from the duality relation (4) that $y_{o}^{*}$ is a measure valued function $\left(y_{o}^{*}=\lambda^{o}\right)$ so that

$$
\tilde{\ell}_{r}(y)=y_{o}^{*}(y)=\int_{I \times B_{r}} y(t, \xi) \lambda_{t}^{o}(d \xi) d t \equiv<y, \lambda^{o}>_{Y_{r}, Y_{r}^{*}}
$$

for all $y \in Y_{r}$. By transposing the isomorphism $L_{r}^{*}$, we obtain

$$
\tilde{\ell}_{r}\left(L_{r}^{*} \eta\right)=\left(L_{r} \lambda^{o}, \eta\right)_{L_{\infty}(I), L_{1}(I)}=\ell_{r}(\eta)=\left(h_{r}, \eta\right)_{L_{\infty}, L_{1}}
$$

for every $\eta \in L_{1}(I)$. This implies the identity $L_{r} \lambda^{o}=h_{r}$ and hence the existence of a sign indefinite measure solution $\lambda_{o} \in L_{\infty}^{w}\left(I, M_{r b a}(E)\right)$ for the original problem (2). Clearly, for every $r(>0)$ finite and $e^{*} \in E^{*}$ and $\nu \in M_{r b a}(E)$ the function $h_{r} \in L_{\infty}(I)$. Thus we may conclude that for any initial (condition) measure $\mu_{0}=\nu \in M_{r b a}(E)$, the corresponding solution $\lambda^{o} \in L_{\infty}^{w}\left(I, M_{r b a}(E)\right)$ and it satisfies the identity (14) for every finite $r \geq 0$ and every $e^{*} \in D\left(A^{*}\right) \subset E^{*}$ and $t \in I$. This completes the proof of existence.

Remark 3.5. The question is: if the initial state $\mu_{0}=\nu$ belongs to $\Pi_{r b a}(E)$ does the corresponding measure solution belong to $L_{\infty}^{w}\left(I, \Pi_{r b a}(E)\right)$. The technique used in [2-11] for proof of existence of measure solutions directly ensures this property. Unfortunately the technique used here does not.

One way to deal with the above problem using the present technique is to verify if the generalized Farkas-like theorem proved by Hernandez-Lerma \& Lasserre in [16, Theorem 2.4, p. 152], and Craven and Koliha in [17, Theorem 1, Theorem 2, p. 987] hold. Let $\mathcal{C} \equiv B C^{+}\left(B_{r}(E)\right)$ denote the positive cone in $B C\left(B_{r}(E)\right)$ with its dual $\mathcal{C}^{*} \equiv M_{r b a}^{+}\left(B_{r}(E)\right)$ contained in $M_{r b a}\left(B_{r}(E)\right)$. Similarly, let $\mathcal{S} \equiv L_{1}(I, \mathcal{C})$ denote the positive cone in $L_{1}\left(I, B C\left(B_{r}(E)\right)\right.$ with the corresponding dual cone $\mathcal{S}^{*} \equiv L_{\infty}^{w}\left(I, \mathcal{C}^{*}\right)$ in $L_{\infty}^{w}\left(I, M_{r b a}\left(B_{r}(E)\right)\right.$. Define the operator $H_{r}: M_{r b a}(E) \longrightarrow L_{\infty}(I)$ by

$$
H_{r}(\nu)(t) \equiv \int_{B_{r}}<S^{*}(t) e^{*}, G(\xi)>_{E^{*}, E} \nu(d \xi) \equiv h_{r}(t), t \in I,
$$


where $G(\xi) \equiv \xi+g(0, \xi), \xi \in E$. In this notation the functional equation (14) is given by

$$
L_{r} \mu=H_{r} \nu
$$

where $\mu_{0}=\nu$ is the initial state (measure). We state the following result giving the necessary and sufficient conditions for existence of a solution $\mu \in L_{\infty}^{w}\left(I, \mathcal{C}^{*}\right)$.

Proposition 3.6. Consider the functional equation $L_{r} \mu=H_{r} \nu$, and the Banach space $Y_{r}$ with the topology introduced in the proof of Theorem 3.4 and suppose $\mathcal{S} \cap Y_{r} \neq \emptyset$. Then, the following two conditions are equivalent:

(i) $H_{r}\left(\mathcal{C}^{*}\right) \subset L_{r}\left(\mathcal{S}^{*} \cap Y_{r}^{*}\right)$

(ii) for $\eta \in L_{1}(I), L_{r}^{*} \eta \in \mathcal{S} \cap Y_{r} \Longrightarrow H_{r}^{*} \eta \in \mathcal{C}$.

Proof. Using the expressions (16)-(18), it is easy to verify that there exists a constant $c>0$ such that $\left\|L_{r} \mu\right\|_{L_{\infty}(I)} \leq c\|\mu\|_{L_{\infty}^{w}\left(I, M_{r b a}\left(B_{r}(E)\right)\right.}$. Thus the operator $L_{r}$ is continuous in the strong topologies of $L_{\infty}^{w}\left(I, M_{r b a}\left(B_{r}(E)\right)\right.$ and $L_{\infty}(I)$ respectively. Then it follows from Dunford and Schwartz [12, Theorem 15 , p. 422] that $L_{r}$ is continuous in the $w^{*}$-topologies of $L_{\infty}^{w}\left(I, M_{r b a}\left(B_{r}(E)\right)\right)$ and $L_{\infty}(I)$ respectively. Using similar arguments one can conclude that $H_{r}$ is also a continuous map in the $w^{*}$-topologies of $M_{r b a}(E)$ and $L_{\infty}(I)$ respectively. Since, by assumption, $\mathcal{S} \cap Y_{r}$ is nonempty we have $\mathcal{S}^{*} \cap Y_{r}^{*}$ is nonempty. Thus, by Theorem 1 of Craven and Koliha [17, Theorem 1, p. 986], it suffices to verify that the set $L_{r}\left(\mathcal{S}^{*} \cap Y_{r}^{*}\right)$ is $w^{*}$-closed. First recall (see Theorem 3.4) that $L_{r}\left(\mathcal{S}^{*} \cap Y_{r}^{*}\right) \subset$ $L_{\infty}(I)$. Let $g_{\alpha} \in L_{r}\left(\mathcal{S}^{*} \cap Y_{r}^{*}\right), \alpha \in D$, be a net that converges in the $w^{*}$-topology to $g_{o} \in L_{\infty}(I)$. We show that $g_{o} \in L_{r}\left(\mathcal{S}^{*} \cap Y_{r}^{*}\right)$. Clearly, there exists a corresponding net $\left\{\mu_{\alpha}\right\} \in \mathcal{S}^{*} \cap Y_{r}^{*}$ so that $L_{r}\left(\mu_{\alpha}\right)=g_{\alpha}$. Since the net $\left\{g_{\alpha}, \alpha \in D\right\}$ is $w^{*}$ convergent it is bounded. Thus there exists a constant $b>0$ such that for all $\alpha \in D$,

$$
\left|\left(L_{r} \mu_{\alpha}, h\right)_{L_{\infty}(I), L_{1}(I)}\right|=\left|\left(g_{\alpha}, h\right)\right| \leq\left\|g_{\alpha}\right\|_{L_{\infty}(I)} \leq b, \forall h \in B_{1}\left(L_{1}(I)\right) .
$$

Hence $\sup _{\alpha \in D}\left|\left(\mu_{\alpha}, L_{r}^{*} h\right)_{Y_{r}^{*}, Y_{r}}\right| \leq b \forall h \in B_{1}\left(L_{1}(I)\right)$ and therefore

$$
\sup _{\alpha \in D}\left|\left(\mu_{\alpha}, y\right)_{Y_{r}^{*}, Y_{r}}\right| \leq b \forall y \in L_{r}^{*}\left(B_{1}\left(L_{1}(I)\right)\right) .
$$

We have seen in Theorem 3.4 (see $(23)$ ) that $Y_{r}=\mathcal{R}\left(L_{r}^{*}\right)$, (range of the operator $\left.L_{r}^{*}\right)$, is a Banach space with respect to the norm topology given there and that $L_{r}^{*} \in \mathcal{I} s o\left(L_{1}, Y_{r}\right)$. Thus it follows from the above inequality that there exists a constant $\bar{b}>0$, possibly dependent on $b$, such that $\sup _{\alpha \in D}\left\|\mu_{\alpha}\right\|_{Y_{r}^{*}} \leq \bar{b}<\infty$ and hence the net $\left\{\mu_{\alpha}\right\}$ is contained in a bounded subset of $Y_{r}^{*}$ which is a subspace of $L_{\infty}^{w}\left(I, M_{r b a}\left(B_{r}(E)\right)\right)$. Since a bounded set in $L_{\infty}^{w}\left(I, M_{r b a}\left(B_{r}(E)\right)\right)$ is relatively $w^{*}$ compact, there exists a subnet of the net $\left\{\mu_{\alpha}\right\}$, relabeled as the original 
net, and a $\mu_{o} \in L_{\infty}^{w}\left(I, M_{r b a}\left(B_{r}(E)\right)\right)$ such that $\mu_{\alpha} \stackrel{w^{*}}{\longrightarrow} \mu_{o}$. Since $\mathcal{S}^{*}$ is a closed (positive) cone in $L_{\infty}^{w}\left(I, M_{r b a}\left(B_{r}(E)\right)\right)$ and $Y_{r}^{*}$ is a Banach space contained in $L_{\infty}^{w}\left(I, M_{r b a}\left(B_{r}(E)\right)\right)$ we have $\mu_{o} \in \mathcal{S}^{*} \cap Y_{r}^{*}$. By $w^{*}$-continuity of the operator $L_{r}$ we have $g_{o}=L_{r}\left(\mu_{o}\right)$ and thus $g_{o} \in L_{r}\left(\mathcal{S}^{*} \cap Y_{r}^{*}\right)$ proving $w^{*}$-closure of the range $L_{r}\left(\mathcal{S}^{*} \cap Y_{r}^{*}\right)$. Considering $w^{*}$-topology, in place of weak topology, it follows from the same procedure as given in the proof of $[17$, Theorem 1, p. 986], that the two conditions are equivalent. This completes the proof.

According to the above proposition, the functional equation (19) has a nonnegative measure solution $\mu$, that is, $\mu \in \mathcal{S}^{*}$, if and only if, $L_{r}^{*} \eta \in \mathcal{S}$ implies $\left(h_{r}, \eta\right) \geq 0$, for any $\eta \in L_{1}(I)$. Among other things, this requires that the set $\Gamma \equiv\left\{\eta \in L_{1}(I): L_{r}^{*} \eta \in \mathcal{S} \cap Y_{r}\right\}$ is nonempty and that, for every $\eta \in \Gamma$, $\ell_{r}(\eta) \equiv\left(h_{r}, \eta\right) \geq 0$.

Remark 3.7 The generalized Farkas-like theorem proved by Hernandez-Lerma \& Lasserre in [16, Theorem 2.4, p. 152] generalizes the results of Craven and Koliha [17] by removing the requirement of closedness of the set $L_{r}\left(S^{*}\right)$. But their results require separability of the Banach space $X$. In our case $X \equiv L_{1}(I, B C(E))$. Since the Banach space $B C(E)$ is not separable the space $X \equiv L_{1}(I, B C(E))$ is not. Hence, even though Theorem 2.4 of [16] generalizes the Theorems 1, 2 of [17], it does not cover nonseparable Banach spaces as considered here.

In the following corollary we prove uniqueness.

Corollary 3.8 (Uniqueness and well poshness). Under the assumptions of Theorem 3.4, the measure valued solution of the functional equation (14), equivalently equation (19), is unique and this solution is continuously dependent on the initial data.

Proof. We prove this by contradiction. Suppose there are two solutions $\mu^{1}, \mu^{2} \in$ $L_{\infty}^{w}\left(I, M_{r b a}(E)\right)$ corresponding to the same initial state $\mu_{0} \in M_{r b a}(E)$. Then it follows from (19) that, for every $r(>0)$ finite, $L_{r}\left(\mu^{1}-\mu^{2}\right)=0$ and so

$$
0=<L_{r}\left(\mu^{1}-\mu^{2}\right), \eta>_{L_{\infty}(I), L_{1}(I)}=<\mu^{1}-\mu^{2}, L_{r}^{*} \eta>_{Y_{r}^{*}, Y_{r}} \quad \forall \eta \in L_{1}(I) .
$$

Since $L_{r}^{*} \in \mathcal{I} s o\left(L_{1}(I), Y_{r}\right)$, this is equivalent to

$$
0=<\mu^{1}-\mu^{2}, y>_{Y_{r}^{*}, Y_{r}} \quad \forall y \in Y_{r} .
$$

Under the canonical embedding $Y_{r} \hookrightarrow Y_{r}^{* *}$, one can consider $Y_{r}$ as a subset of $Y_{r}^{* *}$, that is, $Y_{r} \subset Y_{r}^{* *}$. By virtue of Goldstine's theorem [12, Theorem 5, p. 424], 
the unit ball of $Y_{r}$ is weak star dense in the unit ball of $Y_{r}^{* *}$, that is,

$$
\overline{B_{1}\left(Y_{r}\right)} w^{*}=B_{1}\left(Y_{r}^{* *}\right) .
$$

Thus for every $y^{* *} \in B_{1}\left(Y_{r}^{* *}\right)$, there exists a sequence $y_{n} \in B_{1}\left(Y_{r}\right)$ converging to $y^{* *}$ in the weak star topology. Clearly, $<\mu^{1}-\mu^{2}, y_{n}>_{Y_{r}^{*}, Y_{r}}=0$ for all $n \in N$, and we have

$$
\begin{aligned}
0= & \lim _{n \rightarrow \infty}<\mu^{1}-\mu^{2}, y_{n}>_{Y_{r}^{*}, Y_{r}}=\lim _{n \rightarrow \infty}<\mu^{1}-\mu^{2}, y_{n}>_{Y_{r}^{*}, Y_{r}^{* *}} \\
& =<\mu^{1}-\mu^{2}, y^{* *}>_{Y_{r}^{*}, Y_{r}^{* *}} .
\end{aligned}
$$

Since $Y_{r}^{* *}$ separates points of $Y_{r}^{*}$, and $y^{* *}$ is an arbitrary element of the unit ball $B_{1}\left(Y_{r}^{* *}\right)$, we have $\mu^{1}=\mu^{2}$ as elements of $Y_{r}^{*}$. Thus the solution is unique. For the proof of continuous dependence, let $\left\{\nu^{n}, \nu^{o}\right\} \in M_{r b a}(E)$ denote the initial states and $\left\{\mu^{n}, \mu^{o}\right\} \in L_{\infty}^{w}\left(I, M_{r b a}(E)\right)$ the corresponding (sign indefinite) measure solutions of equation (14) associated with the original system (2). For any finite $r>0$, recall the definition of the operator $\nu \longrightarrow H_{r}(\nu)$ from $M_{r b a}(E)$ to $L_{\infty}(I)$ given by (26). Clearly, $H_{r}$ is a linear operator continuous with respect to the $w^{*}$ topologies on the spaces $M_{r b a}\left(B_{r}(E)\right)$ and $L_{\infty}(I)$ respectively. Then it is easy to verify from the expression $L_{r}\left(\mu^{n}\right)-L_{r}\left(\mu^{o}\right)=L_{r}\left(\mu^{n}-\mu^{o}\right)=H_{r}\left(\nu^{n}-\nu^{o}\right)$ that $\mu^{n} \stackrel{w^{*}}{\longrightarrow} \mu^{o}$ in $L_{\infty}^{w}\left(I, M_{r b a}\left(B_{r}(E)\right)\right)$ as $\nu^{n} \stackrel{w^{*}}{\longrightarrow} \nu^{o}$ in $M_{r b a}\left(B_{r}(E)\right)$ for every $r(>0)$ finite. This completes the proof.

Remark 3.9. It follows from Theorem 3.4 that for any initial measure $\mu_{0} \in$ $M_{r b a}(E)$, the functional equation (14) has at least one sign indefinite measure solution $\mu \in L_{\infty}^{w}\left(I, M_{r b a}(E)\right)$. This allows us to introduce a bounded linear evolution operator $T(t, s), t \geq s \geq 0$, on the Banach space $M_{r b a}(E)$ so that

$$
\mu_{t}=T(t, 0) \mu_{0}, t \geq 0 \text {. }
$$

It follows from uniqueness, as proved in Corollary 3.8, that $T(t, s)$ satisfies the evolution (semigroup) property $T(t, s)=T(t, r) T(r, s), 0 \leq s \leq r \leq t<\infty$.

\section{Optimal FeEdBack CONTROL}

Let $F$ be any real Banach space and $U \subset F$ a weakly compact convex set. Consider the control system

$$
(d / d t)(x+g(t, x))=A x+f(t, x)+B(t) u(t, x), x(0)=x_{0} \in E, t \in I,
$$

where $B$ is a strongly measurable bounded operator valued function with values $B(t) \in \mathcal{L}(F, E)$ and $u$ is the control defined on $I \times E$ taking values in $U$. Let 
$\mathcal{U}_{a d}$ denote the class of admissible feedback controls which consists of functions defined on $I \times E$ and taking values in $U$ so that for each $v \in F^{*}$,

$$
(t, \xi) \longrightarrow<u(t, \xi), v>_{F, F^{*}}
$$

is continuous on every bounded subset of $I \times E$, for example, $I \times B_{r}(E)$ where $B_{r}(E)$ is a closed ball in $E$ of radius $r(>0)$ finite (centered at the origin). This is endowed with the topology of weak convergence in $F$ uniformly on bounded subsets of $I \times E$. We denote this topology by $\tau_{w u b}$. Let $D$ denote a directed set $(D, \geq)$ and $\left\{u^{\alpha}, \alpha \in D\right\}$ a net in $\mathcal{U}_{a d}$. The net is said to converge in this topology to $u^{o} \in \mathcal{U}_{a d}$, denoted by $u^{\alpha} \stackrel{\tau_{\text {wub }}}{\longrightarrow} u^{o}$, if for every $v \in F^{*}$

$$
<v, u^{\alpha}(t, \xi)>_{F^{*}, F} \longrightarrow<v, u^{o}(t, \xi)>_{F^{*}, F} \quad \text { uniformly on } I \times B_{r}(E)
$$

for every finite $r>0$. We assume throughout the rest of the paper that $\mathcal{U}_{a d}$ is compact with respect to the topology $\tau_{w u b}$. In fact, by use of Hahn-Banach separation theorem and the assumption that $U$ is a weakly compact convex set of the Banach space $F$, one can easily verify that $\mathcal{U}_{a d}$ is $\tau_{w u b}$ compact.

Let $L_{r}(u)$ denote the operator $L_{r}$ corresponding to the evolution equation (27) with control $u \in \mathcal{U}_{a d}$. As seen in Theorem 3.4, these operators belong to the space of bounded linear operators, $\mathcal{L}\left(L_{\infty}^{w}\left(I, M_{r b a}(E)\right), L_{\infty}(I)\right)$. Since Theorem 3.4 holds also for the controlled system (27), we can claim that, given the initial (state) measure $\mu_{0} \in \Pi_{r b a}(E)$ and a control $u \in \mathcal{U}_{a d}$, the system (27) has a unique measure valued solution $\mu^{u} \in L_{\infty}^{w}\left(I, \Pi_{r b a}(E)\right) \subset L_{\infty}^{w}\left(I, M_{r b a}^{+}(E)\right)$.

A general control problem is: find a control law $u^{o} \in \mathcal{U}_{a d}$ that extremizes (minimizes or maximizes) the functional

$$
J(u) \equiv G\left(u, \mu^{u}\right)
$$

where $G: \mathcal{U}_{a d} \times L_{\infty}^{w}\left(I, \Pi_{r b a}(E)\right) \longrightarrow \bar{R}$, the extended real number system. To consider such problems we require continuous dependence of solutions with respect to controls. This is proved in the following theorem.

Theorem 4.1. Suppose the assumptions of Theorem 3.4 hold. Let B be a strongly measurable uniformly bounded operator valued function with values in $\mathcal{L}(F, E)$ and $\mathcal{U}_{\text {ad }}$ the class of admissible feedback controls endowed with the $\tau_{\text {wub }}$ topology. Then the measure solution of equation (27) is continuously dependent on control in the sense that whenever $u^{\alpha} \stackrel{\tau_{w u b}}{\longrightarrow} u^{o}$ in $\mathcal{U}_{a d}, \mu^{\alpha} \stackrel{w^{*}}{\longrightarrow} \mu^{o}$ in $L_{\infty}^{w}\left(I, \Pi_{r b a}(E)\right)$ where $\left\{\mu^{\alpha}, \mu^{o}\right\}, \alpha \in D$, are the measure solutions of equation (27) corresponding to the controls $\left\{u^{\alpha}, u^{o}\right\}$ respectively.

Proof. Corresponding to the control system (27), the functional equation (14) is augmented by an additional term associated with the control as follows: 


$$
\begin{aligned}
\int_{B_{r}}<\xi & +g(t, \xi), e^{*}>_{E, E^{*}} \mu_{t}(d \xi)=\int_{B_{r}}<S^{*}(t) e^{*}, \xi+g(0, \xi)>\mu_{0}(d \xi) \\
& +\int_{0}^{t} \int_{B_{r}}<S^{*}(t-s) e^{*}, f(s, \xi)+B(s) u(s, \xi)>\mu_{s}(d \xi) d s \\
& -\int_{0}^{t} \int_{B_{r}}<S^{*}(t-s) A^{*} e^{*}, g(s, \xi)>\mu_{s}(d \xi) d s, t \in I .
\end{aligned}
$$

For a given initial state (measure) $\mu_{0} \in \Pi_{r b a}(E)$, let $\left\{\mu^{\alpha}, \mu^{o}\right\} \in L_{\infty}^{w}\left(I, \Pi_{r b a}(E)\right)$ denote the solutions of the above functional equation corresponding to the controls $\left\{u^{\alpha}, u^{o}\right\} \in \mathcal{U}_{a d}$ respectively. Existence of solution follows from Theorem 3.4 with $f$ replaced by $f+B u$. Introduce the operator $L_{r, 4}$ mapping $L_{\infty}^{w}\left(I, M_{r b a}(E)\right)$ to $L_{\infty}(I)$ as follows

$$
\left(L_{r, 4} \mu\right)(t) \equiv \int_{0}^{t} \int_{B_{r}}<S^{*}(t-s) e^{*}, B(s) u^{o}(s, \xi)>\mu_{s}(d \xi) d s, t \in I,
$$

and define $\hat{L}_{r}=L_{r}+L_{r, 4}$. Then it follows from simple algebraic manipulations, involving the expression (29) corresponding to the controls $\left\{u^{\alpha}, u^{o}\right\}$ and the associated solutions $\left\{\mu^{\alpha}, \mu^{o}\right\}$ respectively, that

$$
\begin{aligned}
& -\hat{L}_{r}\left(\mu^{\alpha}-\mu^{o}\right)(t) \\
& =\int_{0}^{t} \int_{B_{r}}<B^{*}(s) S^{*}(t-s) e^{*}, u^{o}(s, \xi)-u^{\alpha}(s, \xi)>_{F^{*}, F} \mu_{s}^{\alpha}(d \xi) d s, t \in I .
\end{aligned}
$$

Since $U$ is a weakly compact subset of $F$, it is a bounded set in $F$. By our assumption, $B$ is a uniformly bounded operator valued function with values in $\mathcal{L}(F, E)$. Thus it follows from these properties that, for any $e^{*} \in E^{*}$ and any finite $r>0$, the right hand expression of (31) is in $L_{\infty}(I)$ for all $\alpha \in D$. Scalar multiplying the above expression by any $\eta \in L_{1}(I)$ and using Fubini's theorem, we arrive at the following identity

$$
\begin{aligned}
<\hat{L}_{r}\left(\mu^{\alpha}-\mu^{o}\right), \eta>_{L_{\infty}, L_{1}} & =<\mu^{\alpha}-\mu^{o},\left(\hat{L}_{r}\right)^{*} \eta>_{Y_{r}^{*}, Y_{r}} \\
& =-\int_{I \times B_{r}} \psi_{\alpha}(s, \xi) \mu_{s}^{\alpha}(d \xi) d s
\end{aligned}
$$

where

$$
\psi_{\alpha}(s, \xi) \equiv \int_{s}^{T} d t \eta(t)<B^{*}(s) S^{*}(t-s) e^{*}, u^{o}(s, \xi)-u^{\alpha}(s, \xi)>_{F^{*}, F} .
$$


Since $u^{\alpha} \stackrel{\tau_{\text {wub }}}{\longrightarrow} u^{o}$, it follows from the above expression and Lebesgue bounded convergence theorem that $\psi_{\alpha} \rightarrow 0$ strongly in $L_{1}\left(I, B C\left(B_{r}(E)\right)\right)$ for every finite $r>0$. Combining this with the fact that $0 \leq \mu_{s}^{\alpha}\left(B_{r}\right) \leq \mu_{s}^{\alpha}(E) \leq 1$ for all $s \in I$, and $\alpha \in D$, we conclude that the expression on the right hand side of equation (32) converges to zero as $\alpha \rightarrow \infty$. Then it follows from the fact that $\left(\hat{L}_{r}\right)^{*} \in \mathcal{I} s o\left(L_{1}, Y_{r}\right)$ (see Theorem 3.4) that $\mu^{\alpha} \stackrel{w^{*}}{\longrightarrow} \mu^{o}$ in $Y_{r}^{*} \subset L_{\infty}^{w}\left(I, \Pi_{r b a}(E)\right)$. This completes the proof.

Control Problem P1 (Following a Moving Target): Let $c b c(E)$ denote the class of nonempty closed bounded convex subsets of the Banach space $E$ and let $K: I \longrightarrow c b c(E)$ be a multi valued map continuous in the Hausdorff metric. The problem is to find a control (law) such that the distance of the support of the measure solution from the set valued function $K$ is minimized. This can be formulated as the problem of minimizing the functional

$$
J_{1}(u) \equiv \int_{I \times E} d(\xi, K(t)) \mu_{t}^{u}(d \xi) d t
$$

where $d(\xi, K(t))$ denotes the distance of the point $\xi$ from the set $K(t)$.

Theorem 4.2. Consider the control problem (P1) subject to the dynamic system (27) and suppose the assumptions of Theorem 4.1 hold and that there is at least one control for which $J_{1}$ is finite. Then there exists an optimal control $u^{o} \in \mathcal{U}_{\text {ad }}$ at which $J_{1}$ attains its minimum.

Proof. Since the function $\xi \longrightarrow d(\xi, K(t))$ is continuous and bounded on bounded sets but may become unbounded as $|\xi|_{E} \rightarrow \infty$, there may be controls for which the integral will diverge to $+\infty$. If for all $u \in \mathcal{U}_{a d}, J_{1}(u)=\infty$, there is nothing to prove. So, under the assumption that there is at least one control for which the functional is finite, we want to show that there is an optimal one. Consider the weight function $w_{\rho}(\xi) \equiv \exp -\rho\left\{|\xi|_{E}\right\}$ for $\rho>0$. Clearly, this is a continuous symmetric nonnegative real valued function on $E$ and the product $w_{\rho}(\cdot) d(\cdot, K(\cdot)) \in L_{1}(I, B C(E))$. Thus the regularized functional

$$
J_{1, \rho}(u) \equiv \int_{I \times E} w_{\rho}(\xi) d(\xi, K(t)) \mu_{t}^{u}(d \xi) d t
$$

is well defined for all $u \in \mathcal{U}_{a d}$. Hence it follows from Theorem 4.1 that $J_{1, \rho}$ is continuous on $\mathcal{U}_{a d}$ with respect to the $\tau_{w u b}$ topology. Since the admissible set $\mathcal{U}_{a d}$ is $\tau_{w u b}$ compact and $J_{1, \rho}$ is continuous in this topology, it attains its minimum on $\mathcal{U}_{a d}$. Hence for each $\rho>0$, there exists an optimal control $u^{\rho} \in \mathcal{U}_{a d}$ for the regularized problem with the corresponding measure solution $\mu^{\rho} \in L_{\infty}^{w}\left(I, \Pi_{r b a}(E)\right)$. Since there is at least one control for which the original functional $J_{1}$ is finite, 
we can choose a decreasing sequence $\rho_{n} \downarrow 0$ such that $J_{1, \rho_{n}}\left(u^{\rho_{n}}\right)$ is monotone decreasing and it is a minimizing sequence. By virtue of $\tau_{w u b}$ compactness of the set $\mathcal{U}_{a d}$, there exists a generalized subsequence of the sequence $\left\{\rho_{n}\right\}$, relabeled as the original (generalized) sequence $\left\{\rho_{n}\right\}$, and a $u^{o} \in \mathcal{U}_{a d}$ such that $u^{\rho_{n}} \stackrel{\tau_{w u b}}{\longrightarrow} u^{o}$. Let $\left\{\mu^{\rho_{n}}, \mu^{o}\right\}$ denote the corresponding measure solutions of the evolution equation (27). Then by Theorem 4.1, along a further subsequence if necessary, $\mu^{\rho_{n}} \stackrel{w^{*}}{\longrightarrow} \mu^{o}$. Thus taking the limit of the functional (34) along this generalized subsequence we obtain

$$
J_{1}\left(u^{o}\right)=\int_{I \times E} d(\xi, K(t)) \mu_{t}^{o}(d \xi) d t .
$$

This proves the existence.

Control Problem P2 (Concentration of Probability Mass): Let $C$ be a closed subset of $E$ and suppose it is required to find a control policy that maximizes the concentration of (probability) mass on $C$ over the time period $I$. We consider a slightly more general problem. Let $\lambda \in L_{1}^{+}(I)$, a nonnegative integrable function, and consider the objective function

$$
J_{2}(u) \equiv \int_{I} \lambda(t) \mu_{t}^{u}(C) d t
$$

Our problem is to find a control $u \in \mathcal{U}_{a d}$ that maximizes this functional.

Theorem 4.3. Consider the problem (P2) with the objective functional (36) subject to the dynamic system (27). Suppose the assumptions of Theorem 4.1 hold. Then there exists an optimal control $u^{o} \in \mathcal{U}_{\text {ad }}$ maximizing the functional (36).

Proof. We regularize the problem as follows. Let $\varepsilon>0$ (as small as desired) and let $C_{\varepsilon} \equiv\{\xi \in E: d(\xi, C)<\varepsilon\}$ be an open neighborhood of $C$ and $C_{\varepsilon}^{\prime}$ its complement. Clearly $\inf _{\xi \in C, \zeta \in C_{\varepsilon}^{\prime}} d(\xi, \zeta)=\varepsilon>0$. Then it follows from [14, Theorem 1.6, p. 4] that there exists a uniformly continuous function $\psi_{\varepsilon} \in B U C(E) \subset B C(E)$ such that $\psi_{\varepsilon}(\xi)=1$ for $\xi \in C$ and equal to zero outside $C_{\varepsilon}$ and otherwise $0 \leq \psi_{\varepsilon}(\xi) \leq 1$. We consider the regularized problem: find $u \in \mathcal{U}_{a d}$ that maximizes the functional

$$
J_{2, \varepsilon}(u)=\int_{I \times E} \lambda(t) \psi_{\varepsilon}(\xi) \mu_{t}^{u}(d \xi) d t .
$$

The function $\varphi_{\varepsilon}$ given by $\varphi_{\varepsilon}(t, \xi) \equiv \lambda(t) \psi_{\varepsilon}(\xi)$ is an element of $L_{1}(I, B C(E))$ and hence the functional (37) is in the form of the standard duality product. It follows from Theorem 4.1 that the functional $J_{2, \varepsilon}(\cdot)$ is continuous in the $\tau_{w u b}$ topology. 
Since $\mathcal{U}_{a d}$ is $\tau_{w u b}$ compact $J_{2, \varepsilon}(\cdot)$ attains its maximum on $\mathcal{U}_{a d}$. Let $u^{\varepsilon} \in \mathcal{U}_{a d}$ denote any of the maximizing net and $\mu^{\varepsilon} \in L_{\infty}^{w}\left(I, \Pi_{r b a}(E)\right)$ the corresponding net of measure solutions of $(27)$ so that $J_{2, \varepsilon}\left(u^{\varepsilon}\right) \geq J_{2, \varepsilon}(u)$ for all $u \in \mathcal{U}_{a d}$. Again, by virtue of compactness of the set $\mathcal{U}_{a d}$, along a generalized subnet if necessary, $u^{\varepsilon} \stackrel{\tau_{w u b}}{\longrightarrow} u^{o}$ in $\mathcal{U}_{a d}$ as $\varepsilon \downarrow 0$ and by Theorem $4.1, \mu^{\varepsilon} \stackrel{w^{*}}{\longrightarrow} \mu^{o}$ in $L_{\infty}^{w}\left(I, \Pi_{r b a}(E)\right)$ where $\mu^{o}$ is the measure solution of the system (27) corresponding to the control $u^{o}$. Since $\psi_{\varepsilon} \in B U C(E)$ and it converges uniformly to the characteristic function of the set $C$ and the measures $\left\{\mu^{\varepsilon}, \mu^{o}\right\}$ are regular and bounded and $\mu^{\varepsilon} \stackrel{w^{*}}{\longrightarrow} \mu^{o}$ we can let $\varepsilon \downarrow 0$ and obtain

$(38) \lim _{\varepsilon \downarrow 0} J_{2, \varepsilon}\left(u^{\varepsilon}\right)=\lim _{\varepsilon \downarrow 0} \int_{I \times E} \lambda(t) \psi_{\varepsilon}(\xi) \mu_{t}^{\varepsilon}(d \xi) d t=\int_{I} \lambda(t) \mu_{t}^{o}(C) d t=J_{2}\left(u^{o}\right)$.

This proves that the functional $J_{2}$ attains its maximum at $u^{o} \in \mathcal{U}_{a d}$ and hence an optimal control exists.

Control Problem P3 (Exit Time Problem): Let $C_{0}$ be a closed subset of $E$ denoting the support of the initial measure $\mu_{0}$ and suppose it is contained in the interior of a closed ball $B_{\gamma} \subset E$ of radius $\gamma$ such that $\partial B_{\gamma} \cap C_{0}=\emptyset$. Here, we define the exit time to be the first time the support of the measure solution has nonempty intersection with the boundary $\partial \mathcal{O}$ of any open set $\mathcal{O}$ containing $B_{\gamma}$ in its interior. The problem is to find a control so that the first exit time of the support of the corresponding measure solution is maximal. This can be formulated as follows. Since the measure solution of equation (27) is an element of the space $L_{\infty}^{w}\left(I, \Pi_{r b a}(E)\right)$ it may not be $w^{*}$-continuous on $I$. However, the function

$$
t \longrightarrow \nu_{t}^{u}(\cdot) \equiv(1 / t) \int_{0}^{t} \mu_{s}^{u}(\cdot) d s, t \in I,
$$

is a weak star continuous (probability) measure valued function. Thus the functional

$$
J_{3}(u) \equiv \tau_{\gamma}(u) \equiv \inf \left\{t \geq 0: \nu_{t}^{u}\left(B_{\gamma}\right)<1\right\}
$$

is well defined. If the underlying set is empty, we set $\tau_{\gamma}(u)=T$. This is the situation when the support of the measure solution $\mu_{t}^{u}$ remains confined in the closed ball $B_{\gamma}$ for all $t \geq 0$.

Theorem 4.4. Consider the exit time problem (P3) and suppose the assumptions of Theorem 4.1 hold. Then, if $\tau_{\gamma}(u)<T$ for all $u \in \mathcal{U}_{a d}$, there exists a control $u^{o}$ that maximizes the functional $J_{3}(\cdot)$ (and hence the exit time). 
Proof. We prove that $J_{3}$ is upper semi-continuous in the $\tau_{w u b}$ topology. Let $\left\{u^{\alpha}\right\} \in \mathcal{U}_{a d}$, and $\left\{\mu^{\alpha}\right\}$ be the corresponding measure solutions of (27) where $\alpha \in D$ (the directed index set). Since $\mathcal{U}_{a d}$ is $\tau_{w u b}$ compact, there exists an element $u^{o} \in \mathcal{U}_{a d}$, such that, along a subnet if necessary, $u^{\alpha}$ converges to $u^{o}$ in $\tau_{w u b}$ topology. Let $\mu^{o} \in L_{\infty}^{w}\left(I, \Pi_{r b a}(E)\right)$ denote the measure solution of (27) corresponding to $u^{o}$. Then, by Theorem 4.1, along a subnet if necessary, $\mu^{\alpha} \stackrel{w^{*}}{\longrightarrow} \mu^{o}$ in $L_{\infty}\left(I, \Pi_{r b a}(E)\right)$. Since $B_{\gamma}$ is a closed set it follows from a well known result $[14$, Theorem 6.1 , p. 40] that

$$
\varlimsup \nu_{t}^{\alpha}\left(B_{\gamma}\right) \leq \nu_{t}^{o}\left(B_{\gamma}\right), t \in I
$$

By the definition of limsup, for every $\varepsilon \in(0,1)$, there exists an element $\alpha_{\varepsilon} \in D$ such that

$$
\nu_{t}^{\alpha}\left(B_{\gamma}\right) \leq \varlimsup \lim \nu_{t}^{\alpha}\left(B_{\gamma}\right)+\varepsilon \quad \forall \alpha>\alpha_{\varepsilon}, t \in I .
$$

Hence

(42) $\inf \left\{t \geq 0: \nu_{t}^{\alpha}\left(B_{\gamma}\right)<1\right\} \leq \inf \left\{t \geq 0: \varlimsup \nu_{t}^{\alpha}\left(B_{\gamma}\right)+\varepsilon<1\right\} \forall \alpha(\in D)>\alpha_{\varepsilon}$.

Again using (41), one can easily verify that

$$
\inf \left\{t \geq 0: \frac{\alpha}{\lim } \nu_{t}^{\alpha}\left(B_{\gamma}\right)+\varepsilon<1\right\} \leq \inf \left\{t \geq 0: \nu_{t}^{o}\left(B_{\gamma}\right)+\varepsilon<1\right\}
$$

Combining (42) and (43) we arrive at the following inequality,

$$
\inf \left\{t \geq 0: \nu_{t}^{\alpha}\left(B_{\gamma}\right)<1\right\} \leq \inf \left\{t \geq 0: \nu_{t}^{o}\left(B_{\gamma}\right)+\varepsilon<1\right\} \quad \forall \alpha(\in D)>\alpha_{\varepsilon} .
$$

Taking limsup on both sides of the above expression we obtain

$$
\frac{\alpha}{\lim }\left\{\inf \left\{t \geq 0: \nu_{t}^{\alpha}\left(B_{\gamma}\right)<1\right\}\right\} \leq \inf \left\{t \geq 0: \nu_{t}^{o}\left(B_{\gamma}\right)+\varepsilon<1\right\}
$$

Since this holds for arbitrary $\varepsilon \in(0,1)$, it follows from this and the definition of $\tau_{\gamma}(u)$ that

$$
\frac{\alpha}{\lim } \tau_{\gamma}\left(u^{\alpha}\right) \leq \tau_{\gamma}\left(u^{o}\right) .
$$

Thus we have $\varlimsup J_{3}\left(u^{\alpha}\right) \leq J_{3}\left(u^{o}\right)$ proving upper semi-continuity of $J_{3}$. Hence $J_{3}$ attains its maximum on the set $\mathcal{U}_{a d}$, proving the existence of an optimal policy. 
Control Problem P4 (Lagrange Problem): Next we consider the following Lagrange problem. Find a control $u \in \mathcal{U}_{a d}$ that minimizes the cost functional

$$
J_{4}(u)=\int_{I \times E} \ell(t, \xi, u(t, \xi)) \mu_{t}^{u}(d \xi) d t
$$

where $\mu^{u}$ is the measure solution of equation (27) corresponding to the control $u \in \mathcal{U}_{a d}$.

Theorem 4.5. Suppose Theorem 4.1 holds and the following conditions are satisfied:

(A1) $\ell$ is non-negative and lower semicontinuous on $\mathcal{U}_{a d}$ in the sense that whenever the net $u^{\alpha} \stackrel{\tau_{w u b}}{\longrightarrow} u^{o}, \underline{\lim }_{\alpha} \ell\left(t, \xi, u^{\alpha}(t, \xi)\right) \geq \ell\left(t, \xi, u^{o}(t, \xi)\right)$ uniformly on bounded subsets of $I \times E$.

(A2) The integrals $\int_{I \times E} \ell(t, \xi, u(t, \xi)) \mu_{t}^{u}(d \xi) d t$ are well defined for all $u \in \mathcal{U}_{a d}$ with values in $R^{+} \equiv[0, \infty) \cup\{+\infty\}$.

Then there exists an admissible control at which $J$ attains its minimum.

Proof. We prove that $J_{4}$ is lower semi continuous on $\mathcal{U}_{a d}$ with respect to the $\tau_{w u b}$ topology. Let $\left\{u^{\alpha}, \alpha \in D\right\}$ be a net and $u^{o} \in \mathcal{U}_{a d}$ such that $u^{\alpha} \stackrel{\tau_{w u k}}{\longrightarrow} u^{o}$, then by assumption (A1),

$$
\frac{\varliminf_{\alpha}}{\alpha}\left(t, \xi, u^{\alpha}(t, \xi)\right) \geq \ell\left(t, \xi, u^{o}(t, \xi)\right)
$$

uniformly on bounded sets of $I \times E$. Choose any $\gamma \in(0, \infty)$ and define $\ell_{\gamma} \equiv \ell \wedge \gamma$. Clearly, it follows from the previous inequality that

$$
\frac{\lim _{\alpha}}{\ell_{\gamma}}\left(t, \xi, u^{\alpha}(t, \xi)\right) \geq \ell_{\gamma}\left(t, \xi, u^{o}(t, \xi)\right)
$$

uniformly on bounded sets of $I \times E$. By virtue of the basic properties of lim inf, for every $\varepsilon>0$ and every finite positive number $r$, there exists $\alpha_{\varepsilon, r} \in D$ such that for all $\alpha>\alpha_{\varepsilon, r}$

$$
\ell_{\gamma}\left(t, \xi, u^{\alpha}(t, \xi)\right) \geq \lim _{\beta \in D} \ell_{\gamma}\left(t, \xi, u^{\beta}(t, \xi)\right)-\varepsilon, \forall(t, \xi) \in I \times B_{r}(E) .
$$

Integrating the above expression with respect to the product measure $\mu_{t}^{\alpha}(\cdot) \times d t$ on $I \times B_{r}(E)$ we arrive at the following inequality,

$$
\begin{aligned}
& \varepsilon \ell(I)+\int_{I \times B_{r}(E)} \ell_{\gamma}\left(t, \xi, u^{\alpha}(t, \xi)\right) \mu_{t}^{\alpha}(d \xi) d t \\
& \geq \int_{I \times B_{r}(E)}\left\{\frac{\left.\lim _{\beta \in D} \ell_{\gamma}\left(t, \xi, u^{\beta}(t, \xi)\right)\right\} \mu_{t}^{\alpha}(d \xi) d t}{}\right.
\end{aligned}
$$


which holds for all $\alpha>\alpha_{\varepsilon, r}$. Now applying $\underline{\lim }_{\alpha}$ on either side of the above inequality and recalling that, by Theorem $4.1, \mu^{\alpha} \stackrel{w^{*}}{\longrightarrow} \mu^{o}$, we obtain the following inequality

$$
\begin{aligned}
& \varepsilon \ell(I)+\frac{\lim }{\alpha} \int_{I \times B_{r}(E)} \ell_{\gamma}\left(t, \xi, u^{\alpha}(t, \xi)\right) \mu_{t}^{\alpha}(d \xi) d t \\
& \geq \int_{I \times B_{r}(E)}\left\{\frac{\lim }{\beta} \ell_{\gamma}\left(t, \xi, u^{\beta}(t, \xi)\right)\right\} \mu_{t}^{o}(d \xi) d t .
\end{aligned}
$$

Since $\varepsilon(>0)$ is arbitrary and the preceding inequality holds for every $\varepsilon>0$, we have

$$
\begin{aligned}
& \frac{\lim }{\alpha} \int_{I \times B_{r}(E)} \ell_{\gamma}\left(t, \xi, u^{\alpha}(t, \xi)\right) \mu_{t}^{\alpha}(d \xi) d t \\
& \geq \int_{I \times B_{r}(E)}\left\{\frac{\lim }{\beta \in D} \ell_{\gamma}\left(t, \xi, u^{\beta}(t, \xi)\right)\right\} \mu_{t}^{o}(d \xi) d t .
\end{aligned}
$$

Clearly it follows from (47) and (48) that

$$
\frac{\lim }{\alpha} \int_{I \times B_{r}(E)} \ell_{\gamma}\left(t, \xi, u^{\alpha}(t, \xi)\right) \mu_{t}^{\alpha}(d \xi) d t \geq \int_{I \times B_{r}(E)} \ell_{\gamma}\left(t, \xi, u^{o}(t, \xi)\right) \mu_{t}^{o}(d \xi) d t .
$$

By assumption (A2) the integrals are well defined for all admissible controls and the associated measure solutions. Hence letting $\gamma \rightarrow \infty$ and then letting $r \rightarrow \infty$ in the above expression we obtain

$$
\frac{\lim }{\alpha} \int_{I \times E} \ell\left(t, \xi, u^{\alpha}(t, \xi)\right) \mu_{t}^{\alpha}(d \xi) d t \geq \int_{I \times E} \ell\left(t, \xi, u^{o}(t, \xi)\right) \mu_{t}^{o}(d \xi) d t .
$$

This shows that $J_{4}\left(u^{o}\right) \leq \underline{\lim }_{\alpha} J_{4}\left(u^{\alpha}\right)$ proving that it is lower semicontinuous with respect to the $\tau_{w u b}$ topology on $\mathcal{U}_{a d}$. Since $\mathcal{U}_{a d}$ is $\tau_{w u b}$ compact, $J_{4}$ attains its minimum on it. This completes the proof.

Remark 4.6. In this section we have presented the existence of optimal controls for several control problems. We leave the questions of necessary conditions of optimality open for future work.

\section{Acknowledgement}

This work was partially supported by the National Science and Engineering Research Council of Canada under grant no A7101. 


\section{REFERENCES}

[1] H.O. Fattorini, A remark on existence of solutions of infinite dimensional noncompact optimal control problems, SIAM J. Control and Optimization 35 (4) (1997), 1422-1433. doi:10.1137/S036301299528788X

[2] N.U. Ahmed, Measure solutions for semilinear evolution equations with polynomial growth and their optimal controls, Discuss. Math. Differential Inclusions 17 (1997), $5-27$.

[3] N.U. Ahmed, Measure solutions for semilinear systems with unbounded nonlinearities, Nonlinear Analysis 35 (1998), 487-503. doi:10.1016/S0362-546X(97)00699-8

[4] N.U. Ahmed, Relaxed solutions for stochastic evolution equations on Hilbert space with polynomial growth, Publicationes Mathematicae, Debrechen 54 (1-2) (1999), $75-101$.

[5] N.U. Ahmed, Measure solutions for semilinear and quasilinear evolution equations and their optimal control, Nonlinear Analysis 40 (2000), 51-72. doi:10.1016/S0362-546X(97)00699-8

[6] N.U. Ahmed, A general result on measure solutions for semilinear evolution equations, Nonlinear Analysis 42 (2000), 1335-1340. doi:10.1016/S0362-546X(99)00145-5

[7] N.U. Ahmed, Deterministic and stochastic neutral systems on Banach spaces and their optimal Fedback controls, Journal of Nonlinear Systems and Applications (2009), 151-160.

[8] N.U. Ahmed, Measure solutions for evolution equations with discontinuous vector Fields, Nonlinear Functional Analysis \& Applications 9 (3) (2004), 467-484.

[9] N.U. Ahmed, Optimal Stochastic Control of Measure Solutions on Hilbert Space, in Systems, Control, Modeling and Optimization (Edited by F. Ceragioli, A. Dontchev, H. Furuta, K. Marti \& L. Pandolfi), Springer, (Proc. IFIP-TC7 Conference, Turin, Italy, 2005)), U.S., (2006), 1-12.

[10] N.U. Ahmed, Measure valued solutions for stochastic evolution equations on Hilbert space and their feedback control, Discuss. Math. Diff. Incl. Control and Optim. 25 (2005), 129-157. doi:10.7151/dmdico.1061

[11] N.U. Ahmed, Measure solutions for impulsive evolution equations with measurable vector Fields, JMAA 319 (2006), 74-93.

[12] N. Dunford and J.T. Schwartz, Linear Operators, Part 1, Interscience Publishers, Inc., New York, 1958.

[13] A.N. Godunov, Peano's theorem in infinite dimensional Hilbert space is false even in a weakened formulation, Math. Zametki 15 (1974), 467-477.

[14] K.R. Parthasarathy, Probability Measures on Metric Spaces, Academic Press, New York and London, 1967.

[15] J. Dieudonné, Deaux examples déquations différentielles, Acta Sci. Math. Szeged 12B (1950), 38-40. 
[16] O. Hernandez-Lerma and J.B. Lasserre, Cone-constrained linear equations in Banach spaces, J. Convex Analysis 4 (1) (1997), 149-164.

[17] B.D. Craven and J.J. Koliha, Generalization of Farkas' theorem, SIAM J. Math. Anal. 8 (6) (1977). doi:10.1137/0508076

[18] N.U. Ahmed, Some Recent Developments in Systems and Control Theory on Infinite Dimensional Banach Spaces, Part 1 \& 2, Proceedings of the 5th International Conference on Optimization and Control with Applications, (Edited by: K.L. Teo, H. Xu and Y. Zhang), Beijing, China, 2012; Publisher: Springer-Verlag (in Print).

[19] N.U. Ahmed, Optimization and Identification of Systems Governed by Evolution Equations on Banach Spaces, Pitman research Notes in Mathematics Series, 184, Longman Scientific and Technical, U.K; Co-published with John-Wiely \& Sons, Inc. New York, 1988.

Received 1 April 2013 
